\title{
Soft Minimal Soft Sets and Soft Prehomogeneity in Soft Topological Spaces
}

\section{Samer Al Ghour}

Department of Mathematics and Statistics, Jordan University of Science and Technology, Irbid, Jordan

\section{]jfis}

\begin{abstract}
In this paper, we give characterizations for soft minimal soft open sets in terms of the soft closure operator, and we conclude that soft subsets of soft minimal soft open sets are soft preopen sets. In addition to these, we define soft minimal soft sets and soft minimal soft preopen sets as two new classes of soft sets in soft topological spaces, and we define soft prehomogeneity as a new soft topological property. We give several relationships regarding these new notions and related known soft topological notions. We show that soft minimal soft preopen sets are soft points, and we prove that soft minimal soft sets with non-null soft interiors are soft minimal soft open sets. Moreover, we show that soft prehomogeneous soft topological space that has a soft minimal soft set is soft locally indiscrete. Also, we give several characterizations of soft locally indiscrete soft topological space in terms of soft minimal soft open sets, soft minimal soft sets, soft preopen sets, and soft prehomogeneity. We deal with correspondence between our new soft topological notions and their analogs topological ones. Finally, we raise six open questions.
\end{abstract}

Keywords: Soft minimal soft open sets, Soft preopen soft sets, Soft locally indiscrete, Prehomogeneity, Generated soft topology

Received: Jul. 4, 2021

Revised : Aug. 16, 2021

Accepted: Aug. 28, 2021

Correspondence to: Samer Al Ghour (algore@just.edu.jo)

(CThe Korean Institute of Intelligent Systems

cCThis is an Open Access article distributed under the terms of the Creative Commons Attribution Non-Commercial License (http://creativecommons.org/licenses/by-nc / 3.0/) which permits unrestricted noncommercial use, distribution, and reproduction in any medium, provided the original work is properly cited.

\section{Introduction}

This paper follows the notions and terminologies as appeared in [1] and [2]. In this paper, TS and STS will denote topological space and soft topological space, respectively. Molodtsov [3] defined soft sets in 1999. The soft set theory offers a general mathematical tool for dealing with uncertain objects. Let $U$ be a universal set and $E$ be a set of parameters. A soft set over $U$ relative to $E$ is a function $G: E \longrightarrow \mathcal{P}(U)$. SS $(U, E)$ will denote the family of all soft sets over $U$ relative to $E$. In this paper, the null soft set and the absolute soft set will be denoted by $0_{E}$ and $1_{E}$, respectively. The structure of STSs was defined in [4] as follows: An STS is a triplet $(U, \tau, E)$, where $\tau \subseteq S S(U, E), \tau$ contains $0_{E}$ and $1_{E}, \tau$ is closed under finite soft intersection, and $\tau$ is closed under arbitrary soft union. Let $(U, \tau, E)$ be an STS and $F \in S S(X, A)$, then $F$ is said to be a soft open set in $(U, \tau, E)$ if $F \in \tau$ and $F$ is said to be a soft closed set in $(U, \tau, E)$ if $1_{A}-F$ is a soft open set in $(U, \tau, E)$. The family of all soft closed sets in $(U, \tau, E)$ will be denoted by $\tau^{c}$. Soft topological concepts and their applications are still a hot area of research [1|2[5-|26]. The concepts of minimal open sets in TSs were defined and investigated in [27]. Then research via this concept is continued by various researchers [28-31]. Author in [32] defined and investigated the concepts of minimal 
sets and minimal preopen sets, in which he also introduced the notion of prehomogeneity. Soft minimal soft open sets in STSs were defined in [33], then research via them is continued in [1] and others.

In this paper, we give characterizations for soft minimal soft open sets in terms of the soft closure operator, and we conclude that soft subsets of soft minimal soft open sets are soft preopen sets. In addition to these, we define soft minimal soft open sets and soft minimal soft preopen sets as two new classes of soft sets in STSs, and we define soft prehomogeneity. as a new soft topological property. We give several relationships regarding these new notions and related known soft topological notions. We show that soft minimal soft preopen sets are soft points and we prove that soft minimal soft sets with non-null soft interiors are soft minimal soft open sets. Moreover, we show that soft prehomogeneous STS that has a soft minimal soft set is soft locally indiscrete. Also, we give several characterizations of soft locally indiscrete in terms of soft minimal soft open sets, soft minimal soft sets, soft preopen sets, and soft prehomogeneity. We deal with correspondence between our new soft topological notions and their analogs topological ones. Finally, we raise six open questions.

\section{Preliminaries}

Herein, we recall several related definitions and results.

Let $(X, \Im)$ be a TS and let $S \subseteq X$. Then $\Im^{c}$ will denote the family of all closed sets in $(X, \Im), C l_{\Im}(S)\left(\operatorname{resp} . \operatorname{Int}_{\Im}(S)\right)$ will denote the closure of $S$ in $(X, \Im)$ (resp. the interior of $S$ in $(X, \Im)$ ), and $M_{(x, \Im)}$ will denote the set $\cap\{V \in \Im: x \in V\}$.

Definition 2.1. Let $(X, \Im)$ be a TS and let $S \subseteq X$. Then $S$ is said to be

(a) [34] a preopen set in $(X, \Im)$ if $S \subseteq \operatorname{Int}_{\Im}\left(C l_{\Im}(S)\right)$. Equivalently: $S$ is a preopen set in $(X, \Im)$ if and only if there exists $V \in \Im$ such that $S \subseteq V \subseteq C l_{\Im}(S)$.

(b) [27] a minimal open set in $(X, \Im)$ if for all $V \in \Im$ with $V \subseteq S$ either $V=\emptyset$ or $V=S$.

(c) [32] a minimal set in $(X, \Im)$ if there exists $x \in X$ such that $S=M_{(x, \Im)}$.

(d) [32] a minimal preopen set in $(X, \Im)$ if $S$ is a preopen set in $(X, \Im)$ and for all preopen set $V$ in $(X, \Im)$ with $V \subseteq S$ either $V=\emptyset$ or $V=S$.

The family of all preopen sets (resp. minimal open sets, minimal sets, minimal preopen sets) in $(X, \Im)$ will be denoted by $P O(X, \Im)$ (resp. $\min (X, \Im), \min s(X, \Im), \min (P O(X, \Im))$ ).
Definition 2.2 [34]. A function $f:(X, \Im) \rightarrow(Y, \aleph)$ is said to be

(a) preirresolute if $f^{-1}(V) \in P O(X, \Im)$ for all $V \in P O(Y, \aleph)$.

(b) prehomeomorphism if $f$ is a bijection and both $f$ and $f^{-1}$ are preirresolute.

Definition 2.3 27]. A TS $(X, \Im)$ is called prehomogeneous if for any $x, y \in X$, there exists a prehomeomorphism $f$ : $(X, \Im) \longrightarrow(X, \Im)$ such that $f(x)=y$.

Definition 2.4 [35]. Let $M, N \in S S(X, A)$.

(a) $M$ is a soft subset of $N$, denoted by $M \widetilde{\simeq} N$, if $M(a) \subseteq$ $N(a)$ for each $a \in A$.

(b) $M$ and $N$ are said to be soft equal, denoted by $F=G$, if $M \widetilde{\subseteq} N$ and $N \widetilde{\subseteq} M$.

(c) Soft union of $M$ and $N$ is denoted by $M \widetilde{\cup} N$ and defined to be the soft set $M \widetilde{\cup} N \in S S(X, A)$ where $(M \widetilde{\cup} N)(a)=$ $M(a) \cup N(a)$ for each $a \in A$.

(d) Soft intersection of $M$ and $N$ is denoted by $M \widetilde{\cap} N$ and defined to be the soft set $M \widetilde{\cap} N \in S S(X, A)$ where $(M \widetilde{\cap} N)(a)=M(a) \cap N(a)$ for each $a \in A$.

(e) The difference of $M$ and $N$ is denoted by $M-N$ and defined to be the soft set $M-N \in S S(X, A)$ where

$(M-N)(a)=M(a)-N(a)$ for each $a \in A$.

Definition $2.5[36]$. Let $\Delta$ be an arbitrary indexed set and $\left\{G_{\alpha}: \alpha \in \Delta\right\} \subseteq S S(X, A)$.

(a) The soft union of these soft sets is the soft set denoted by $\widetilde{\cup}_{\alpha \in \Delta} G_{\alpha}$ and defined by $\left(\underset{\alpha \in \Delta}{\widetilde{\cup}_{\alpha}} G_{\alpha}\right)(a)=\cup_{\alpha \in \Delta} G_{\alpha}(a)$ for each $a \in A$.

(b) The soft intersection of these soft sets is the soft set denoted by $\widetilde{\cap} G_{\alpha \in \Delta}$ and defined by $\left(\widetilde{\cap} G_{\alpha \in \Delta}\right)(a)=\underset{\alpha \in \Delta}{\bigcap} G_{\alpha}(a)$ for each $a \in A$.

Definition 2.6 [1]. Let $X$ be a universal set and $A$ is a set of parameters. Then $G \in S S(X, A)$ defined by

$$
G(a)= \begin{cases}Y, & \text { if } a=e \\ \emptyset, & \text { if } a \neq e\end{cases}
$$

will be denoted by $e_{Y}$.

Definition 2.7 [37]. Let $X$ be a universal set and $A$ be a set of parameters. Then $G \in S S(X, A)$ defined by

$$
G(a)= \begin{cases}\{x\}, & \text { if } a=e, \\ \emptyset, & \text { if } a \neq e\end{cases}
$$

will be denoted by $e_{x}$ and will be called a soft point. 
Definition 2.8 [37]. Let $G \in S S(X, A)$ and $a_{x} \in S P(X, A)$. Then $a_{x}$ is said to belong to $F$ (notation: $a_{x} \widetilde{\in} G$ ) if $a_{x} \widetilde{\subseteq} G$ or equivalently: $a_{x} \widetilde{\in} G$ if and only if $x \in G(a)$.

Theorem $2.9[4]$. Let $(X, \tau, A)$ be an STS. Then the collection $\{F(a): F \in \tau\}$ defines a topology on $X$ for every $a \in A$. This topology will be denoted by $\tau_{a}$.

Theorem 2.10 [38]. Let $(X, \Im)$ be a TS. Then the collection

$$
\{F \in S S(X, A): F(a) \in \Im \text { for all } a \in A\}
$$

defines a soft topology on $X$ relative to $A$. This soft topology will be denoted by $\tau(\Im)$.

Let $(X, \tau, A)$ be an STS and let $F \in S S(X, A)$. Then $C l_{\tau}(F)$ (resp. $\operatorname{Int}_{\tau}(F)$ ) will denote the soft closure of $F$ in $(X, \tau, A)$ (resp. the soft interior of $F$ in $(X, \tau, A)$ ).

Definition 2.11. Let $(X, \tau, A)$ be a TS and let $F \in S S(X, A)$. Then $F$ is said to be

(a) [39] a soft preopen set in $(X, \tau, A)$ if $F \subseteq \operatorname{Int}_{\tau}\left(C l_{\tau}(F)\right)$. Equivalently: $F$ is a soft preopen set in $(X, \tau, A)$ if and only if there exists $G \in \tau$ such that $F \subseteq G \subseteq C l_{\tau}(F)$.

(b) [33] a soft minimal soft open set in $(X, \tau, A)$ if for all $G \in \Im$ with $G \widetilde{\subseteq} F$ either $G=0_{A}$ or $G=F$.

The family of all soft preopen sets (resp. soft minimal soft open sets) in $(X, \tau, A)$ will be denoted by $P O(X, \tau, A)$ (resp. $\min (X, \tau, A))$.

Definition 2.12 [40]. An STS $(X, \tau, A)$ is said to be soft locally indiscrete if $\tau=\tau^{c}$.

Definition 2.13. A soft mapping $f_{p u}:(X, \tau, A) \longrightarrow(Y, \sigma, B)$ is said to be

(a) soft preirresolute if $f_{p u}^{-1}(F) \in P O(X, \tau, A)$ for all $F \in$ $P O(Y, \sigma, B)$.

(b) soft prehomeomorphism if $f_{p u}$ is bijective, and $f_{p u}$ : $(X, \tau, A) \longrightarrow(Y, \sigma, B)$ and $f_{p^{-1} u^{-1}}:(Y, \sigma, B) \longrightarrow(X, \tau, A)$ are soft preirresolute.

\section{Soft Minimal Soft Open Sets, Soft Minimal Soft Sets and Soft Minimal Soft Preopen Sets}

We start this section by the following two characterizations of soft minimal soft open sets:

Theorem 3.1. Let $(X, \tau, A)$ and let $F \in S S(X, A)-\left\{0_{A}\right\}$. Then the following are equivalent:

(a) $F \in \min (X, \tau, A)$.

(b) For every $G \in S S(X, A)-\left\{0_{A}\right\}$ with $G \widetilde{\subseteq} F, F \widetilde{\subseteq} C l_{\tau}(G)$. (c) For every $G \in S S(X, A)-\left\{0_{A}\right\}$ with $G \widetilde{\subseteq} F, C l_{\tau}(G)=$ $C l_{\tau}(F)$.

Proof. (a) $\Longrightarrow$ (b) Let $G \in S S(X, A)-\left\{0_{A}\right\}$ with $G \widetilde{\subseteq} F$. Let $a_{x} \widetilde{\in} F$ and let $M \in \tau$ such that $a_{x} \widetilde{\in} M$, then $F \widetilde{\cap} M \neq$ $0_{A}$ and so $F \widetilde{\subseteq} M$. Thus, $0_{A} \neq G=G \widetilde{\cap} M$. Therefore, $a_{x} \widetilde{\in} C l_{\tau}(G)$.

(b) $\Longrightarrow$ (c) Let $G \in S S(X, A)-\left\{0_{A}\right\}$ with $G \widetilde{\subseteq} F$. Then $C l_{\tau}(G) \widetilde{\simeq} C l_{\tau}(F)$. On the other hand, by (b) we have $F \widetilde{\subseteq} C l_{\tau}$ $(G)$, and so $C l_{\tau}(F) \widetilde{\subseteq} C l_{\tau}\left(C l_{\tau}(G)\right)=C l_{\tau}(G)$. Thus, $C l_{\tau}(G)$ $=C l_{\tau}(F)$.

(c) $\Longrightarrow$ (a) Suppose to the contrary that there exists $G \in \tau-$ $\left\{0_{A}\right\}$ such that $G \widetilde{\subseteq} F$ and $G \neq F$. Choose $a_{x} \widetilde{\in} F-G$. Then we have $a_{x} \widetilde{\in}\left(1_{A}-G\right) \in \tau^{c}$ and so $C l_{\tau}\left(a_{x}\right) \widetilde{\subseteq} C l_{\tau}\left(1_{A}-G\right)=$ $1_{A}-G$. On the other hand, by (c), $C l_{\tau}\left(a_{x}\right)=C l_{\tau}(F)=$ $C l_{\tau}(G)$, a contradiction.

Theorem 3.2. Let $(X, \tau, A)$ and let $F \in \min (X, \tau, A)$. Then for every $G \in S S(X, A)-\left\{0_{A}\right\}$ with $G \widetilde{\subseteq} F$, we have $G \in P O(X, \tau, A)$.

Proof. Let $F \in \min (X, \tau, A)$ and $G \in S S(X, A)-\left\{0_{A}\right\}$ with $G \widetilde{\subseteq} F$. Then by Theorem 3.1, $G \widetilde{\subseteq} F \widetilde{\subseteq} C l_{\tau}(G)$. Hence $G \in P O(X, \tau, A)$.

Theorem 3.3. For an STS $(X, \tau, A)$, the following are equivalent:
(a) $(X, \tau, A)$ is soft locally indiscrete.
(b) $P O(X, \tau, A)=S S(X, A)$.
(c) $S P(X, A) \subseteq P O(X, \tau, A)$.
(d) $\tau^{c} \subseteq P O(X, \tau, A)$.

Proof. (a) $\Longrightarrow$ (b) We need only to show that $S S(X, A) \subseteq$ $P O(X, \tau, A)$. Let $F \in S S(X, A)$, then $C l_{\tau}(F) \in \tau^{c}$. So by (a), $C l_{\tau}(F) \in \tau$. Since $F \widetilde{\subseteq} C l_{\tau}(F) \widetilde{\widetilde{C}} C l_{\tau}(F)$, then $F \in$ $P O(X, \tau, A)$.

(b) $\Longrightarrow$ (c) Since $S P(X, A) \subseteq S S(X, A)$, then by (b) $S P(X, A) \subseteq P O(X, \tau, A)$.

(c) $\Longrightarrow$ (d) Let $G \in \tau^{c}$. Let $a_{x} \widetilde{\in} G$. By (c), $a_{x} \in P O(X, \tau, A)$ and so, $a_{x} \widetilde{\subseteq} I n t_{\tau}\left(C l_{\tau}\left(a_{x}\right)\right) \widetilde{\subseteq} \operatorname{Int}_{\tau}\left(C l_{\tau}(G)\right)$. Hence, $a_{x} \widetilde{\in} I n t_{\tau}$ $\left(C l_{\tau}(G)\right)$. Therefore, $G \widetilde{\subseteq} \operatorname{Int}_{\tau}\left(C l_{\tau}(G)\right)$. Thus, $G \in P O(X$, $\tau, A)$.

(d) $\Longrightarrow$ (a) It is sufficient to show that $\tau^{c} \subseteq \tau$. Let $G \in \tau^{c}$, then by (d), $G \widetilde{\subseteq} I n t_{\tau}\left(C l_{\tau}(G)\right)=\operatorname{Int}_{\tau}(G)$. But $I n t_{\tau}(G) \widetilde{\subseteq} G$ is always true. It follows that $\operatorname{Int}_{\tau}(G)=G$ and hence $G \in$ $\tau$.

Let $(X, \tau, A)$ be an STS. For each $a_{x} \in S P(X, A)$, denote the soft set $\widetilde{\bigcap}\left\{F: F \in \tau\right.$ and $\left.a_{x} \widetilde{\in} F\right\}$ by $M_{\left(a_{x}, \tau\right)}$.

Definition 3.4. Let $(X, \tau, A)$ be an STS and $G \in S S(X, A)$, then $G$ is called a soft minimal soft set in $(X, \tau, A)$ if $G=$ 
$M_{\left(a_{x}, \tau\right)}$ for some $a_{x} \in S P(X, A)$. The family of all soft minimal soft sets in $(X, \tau, A)$ will be denoted by $\operatorname{minss}(X, \tau, A)$.

Theorem 3.5. Let $(X, \tau, A)$ be an STS and $G \in \min (X, \tau, A)$, then for every $a_{x} \widetilde{\in} G, G=M_{\left(a_{x}, \tau\right)}$.

Proof. Suppose that $G \in \min (X, \tau, A)$ and let $a_{x} \tilde{\in} G$. Then by definition of $M_{\left(a_{x}, \tau\right)}$ we have $M_{\left(a_{x}, \tau\right)} \widetilde{\widetilde{\subseteq}} G$. On the other hand, to see that $G \widetilde{\subseteq} M_{\left(a_{x}, \tau\right)}$, suppose to the contrary that there is $b_{y} \widetilde{\in} G-M_{\left(a_{x}, \tau\right)}$. Then $b_{y} \widetilde{\in} G$ and there exists $F \in \tau$ such that $a_{x} \widetilde{\in} F$ and $b_{y} \widetilde{\in} 1_{A}-F$. Since $a_{x} \widetilde{\in} G \widetilde{\cap} F \in \tau$ and $G \in$ $\min (X, \tau, A)$, then $G \widetilde{\subseteq} F$ and hence $b_{y} \widetilde{\in} F$, a contradiction.

Corollary 3.6. For any $\operatorname{STS}(X, \tau, A), \min (X, \tau, A) \subseteq$ $\operatorname{minss}(X, \tau, A)$.

The following example will show that the inclusion in Corollary 3.6 is not equality, in general:

Example 3.7. Let $X=\mathbb{R}, A=\{a, b\}$ and

$$
\tau=\left\{F \in S S(X, A): F(t) \in \Im_{u} \text { for every } t \in A\right\}
$$

For every $n \in \mathbb{N}$, let $F_{n} \in S S(X, A)$ defined by $F_{n}(a)=$ $\left(\frac{-1}{n}, \frac{1}{n}\right)$ and $F_{n}(b)=\emptyset$. Since $a_{0} \widetilde{\in} M_{\left(a_{0}, \tau\right)} \subseteq \widehat{\bigcap_{n \in \mathbb{N}}} F_{n}=a_{0}$, then $M_{\left(a_{0}, \tau\right)}=a_{0}$. But $M_{\left(a_{0}, \tau\right)}$ not even soft preopen.

The following question is natural:

Question 3.8. For an $\operatorname{STS}(X, \tau, A)$, is it true that $\operatorname{minss}(X$, $\tau, A) \cap \tau \subseteq \min (X, \tau, A)$ ?

The following example is a negative answer for Question 3.8:

Example 3.9. Let $X=\{1,2,3\}, A=\{a, b\}$ and $\tau=\{F \in$ $S S(X, A): F(t) \in\{\emptyset, X,\{1\},\{1,2\}\}$ for every $t \in A\}$.

If $b_{2} \widetilde{\in} F \in \tau$, then $2 \in F(b) \in\{\emptyset, X,\{1\},\{1,2\}\}$, and so either $F(b)=X$ or $F(b)=\{1,2\}$. Then $M_{b_{2}}(b)=\{1,2\}$ and $M_{\left(b_{2}, \tau\right)}(a)=\emptyset$, and hence $M_{\left(b_{2}, \tau\right)} \in \operatorname{minss}(X, \tau, A) \cap \tau$. On the other hand, since $b_{1} \in \tau-\left\{0_{A}\right\}$ with $b_{1} \widetilde{\subseteq} M_{\left(b_{2}, \tau\right)}$ and $b_{1} \neq M_{\left(b_{2}, \tau\right)}$, then $M_{\left(b_{2}, \tau\right)} \notin \min (X, \tau, A)$.

The following lemma will be used in the following main result:

Lemma 3.10. Let $(X, \tau, A)$ be a soft locally indiscrete STS. Then

(a) $\operatorname{minss}(X, \tau, A) \subseteq \tau$.

(b) If $M_{\left(a_{x}, \tau\right)} \widetilde{\widetilde{\subseteq}} M_{\left(b_{y}, \tau\right)}$, then $b_{y} \widetilde{\in} M_{\left(a_{x}, \tau\right)}$.

(c) If $M_{\left(a_{x}, \tau\right)} \widetilde{\subseteq} M_{\left(b_{y}, \tau\right)}$, then $M_{\left(a_{x}, \tau\right)}=M_{\left(b_{y}, \tau\right)}$.

Proof. (a) Let $a_{x} \in S P(X, A)$. Since $(X, \tau, A)$ is soft locally indiscrete, then $M_{\left(a_{x}, \tau\right)}=\widetilde{\cap}\left\{F \in \tau^{c}: a_{x} \widetilde{\in} F\right\}$ and so $M_{\left(a_{x}, \tau\right)} \in \tau^{c}$. Then again by soft local indiscreetness of $(X, \tau, A)$, we have $M_{\left(a_{x}, \tau\right)} \in \tau$. (b) Suppose to the contrary that $M_{\left(a_{x}, \tau\right)} \widetilde{\subseteq} M_{\left(b_{y}, \tau\right)}$ and $b_{y} \widetilde{\in} 1_{A}$ $-M_{\left(a_{x}, \tau\right)}$. By (a), $1_{A}-M_{\left(a_{x}, \tau\right)} \in \tau$ and so $M_{\left(b_{y}, \tau\right)} \widetilde{\widetilde{\subseteq}} 1_{A}-$ $M_{\left(a_{x}, \tau\right)}$. Thus, $M_{\left(b_{y}, \tau\right)} \widetilde{\cap} M_{\left(a_{x}, \tau\right)}=0_{A}$. On the other hand, since $M_{\left(a_{x}, \tau\right)} \widetilde{\widetilde{\subseteq}} M_{\left(b_{y}, \tau\right)}$, then $M_{\left(b_{y}, \tau\right)} \widetilde{\cap} M_{\left(a_{x}, \tau\right)}=M_{\left(a_{x}, \tau\right)} \neq$ $0_{A}$, a contradiction.

(c) Suppose that $M_{\left(a_{x}, \tau\right)} \widetilde{\subseteq} M_{\left(b_{y}, \tau\right)}$, then by (b) $b_{y} \widetilde{\in} M_{\left(a_{x}, \tau\right)}$ and so by (a), $M_{\left(b_{y}, \tau\right)} \subseteq \widetilde{\subseteq}_{\left(a_{x}, \tau\right)}$. It follows that $M_{\left(a_{x}, \tau\right)}=$ $M_{\left(b_{y}, \tau\right)}$.

Theorem 3.11. For an STS $(X, \tau, A)$, the following are equivalent:

(a) $(X, \tau, A)$ is soft locally indiscrete.

(b) $\operatorname{minss}(X, \tau, A)=\min (X, \tau, A)$.

(c) $\min (X, \tau, A)$ is a soft base of $(X, \tau, A)$.

(d) $(X, \tau, A)$ has a soft base which forms a soft partition of $1_{A}$.

Proof. (a) $\Longrightarrow$ (b) By Corollary 3.6 we need only to show that $\operatorname{minss}(X, \tau, A) \subseteq \min (X, \tau, A)$. Let $a_{x} \in S P(X, A)$ and let $G \in \tau-\left\{0_{A}\right\}$ with $G \widetilde{\subseteq} M_{\left(a_{x}, \tau\right)}$. Choose $b_{y} \in G$, then $M_{\left(b_{y}, \tau\right)} \widetilde{\widetilde{\subseteq}} G \widetilde{\subseteq} M_{\left(a_{x}, \tau\right)}$. So Lemma 3.10(c), $M_{\left(b_{y}, \tau\right)}=G=$ $M_{\left(a_{x}, \tau\right)}$. This ends the proof.

(b) $\Longrightarrow$ (c) By (b), $\widetilde{\cup}\{F: F \in \min (X, \tau, A)\}=1_{A}$. Thus by Theorem 4.11 of [1], $\min (X, \tau, A)$ is a soft base of $(X, \tau, A)$.

(c) $\Longrightarrow$ (d) By (c) and Theorem 4.11 of [1], $\min (X, \tau, A)$ is a soft base of $(X, \tau, A)$ which forms a soft partition of $1_{A}$.

(d) $\Longrightarrow$ (a) Let $\mathcal{B}$ be a soft base which forms a soft partition of $1_{A}$. Let $G \in \tau-\left\{0_{A}\right\}$, then there exists $\mathcal{B}_{1} \subseteq \mathcal{B}$ such that $\widetilde{\cup}\left\{F: F \in \mathcal{B}_{1}\right\}=G$ and so $1_{A}-G=\widetilde{\cup}\left\{1_{A}-F: F \in \mathcal{B}\right.$ $\left.-\mathcal{B}_{1}\right\} \in \tau$. This shows $G \in \tau^{c}$. Therefore, $(X, \tau, A)$ is soft locally indiscrete.

Definition 3.12. Let $(X, \tau, A)$ be an STS. A soft set $F \in$ $S S(X, A)$ is said to be a soft minimal soft preopen set of $(X, \tau, A)$ if $F \in P O(X, \tau, A)$ and for all $G \in P O(X, \tau, A)$ with $G \widetilde{\subseteq} F$ either $G=0_{A}$ or $G=F$. The family of all soft preopen sets in $(X, \tau, A)$ will be denoted by $\min (P O(X, \tau, A))$.

The following lemma will be necessary for proving the next main result:

Lemma 3.13. Let $(X, \tau, A)$ be an STS. If $F \in \tau$ and $G \in$ $P O(X, \tau, A)$, then $F \widetilde{\cap} G \in P O(X, \tau, A)$.

Proof. Let $F \in \tau$ and $G \in P O(X, \tau, A)$. Since $G \in$ $P O(X, \tau, A)$, then there exists $K \in \tau$ such that $G \widetilde{\subseteq} K \widetilde{\subseteq} C l_{\tau}(G)$ and so $F \widetilde{\cap} G \widetilde{\subseteq} F \widetilde{\cap} K \widetilde{\subseteq} F \widetilde{\cap} C l_{\tau}(G)$. Since $F \in \tau$, then $F \widetilde{\cap}$ $C l_{\tau}(G) \widetilde{\simeq} C l_{\tau}(F \widetilde{\cap} G)$. Hence, $F \widetilde{\cap} G \in P O(X, \tau, A)$.

The following theorem shows that soft minimal soft preopen sets are the soft points soft preopen sets: 
Theorem 3.14. For any $\operatorname{STS}(X, \tau, A), \min (P O(X, \tau, A))=$ $P O(X, \tau, A) \cap S P(X, A)$.

Proof. By the definition of $P O(X, \tau, A), \min (P O(X, \tau, A))$ $\subseteq P O(X, \tau, A)$. To show that $\min (P O(X, \tau, A)) \subseteq S P(X, A)$, let $F \in \min (P O(X, \tau, A))$. Choose $a_{x} \tilde{\in} F$. We are going to show that $a_{x} \in P O(X, \tau, A)$. By Lemma 3.13,

$$
\left(1_{A}-C l_{\tau}\left(a_{x}\right)\right) \widetilde{\cap} F \in P O(X, \tau, A) .
$$

Since $F \in \min (P O(X, \tau, A)),\left(1_{A}-C l_{\tau}\left(a_{x}\right)\right) \widetilde{\cap} F \widetilde{\subseteq} F$, and $\left(1_{A}-C l_{\tau}\left(a_{x}\right)\right) \widetilde{\cap} F \neq F$, then $\left(1_{A}-C l_{\tau}\left(a_{x}\right)\right) \widetilde{\cap} F=0_{A}$ and so $F \widetilde{\subseteq} C l_{\tau}\left(a_{x}\right)$. Hence, $C l_{\tau}(F) \widetilde{\subseteq} C l_{\tau}\left(a_{x}\right)$ and thus $a_{x} \widetilde{\subseteq} F \widetilde{\subseteq}$ $\operatorname{Int}_{\tau}\left(C l_{\tau}(F)\right) \widetilde{\subseteq} \operatorname{Int}_{\tau}\left(C l_{\tau}\left(a_{x}\right)\right) \widetilde{\subseteq} C l_{\tau}\left(a_{x}\right)$. Therefore, $a_{x} \in$ $P O(X, \tau, A)$. Now since $F \in \min (P O(X, \tau, A))$, then $F=$ $a_{x}$. It follows that $F \in S P(X, A)$. Conversely, it is clear that $P O(X, \tau, A) \cap S P(X, A) \subseteq \min (P O(X, \tau, A))$.

Theorem 3.15. If $F \in \min (X, \tau, A)$, then $\left\{a_{x}: a_{x} \widetilde{\in} F\right\} \subseteq$ $\min (P O(X, \tau, A))$.

Proof. Let $F \in \min (X, \tau, A)$ and let $a_{x} \widetilde{\in} F$, then by Theorem 3.2 we have $a_{x} \in P O(X, \tau, A)$. Thus, $a_{x} \in P O(X, \tau, A)$ $\cap S P(X, A)$. Hence, by Theorem 3.14 we have

$$
a_{x} \in \min (P O(X, \tau, A)) .
$$

The following example shows that the implication in Theorem 3.15 is not reversible, in general:

Example 3.16. Let $X=\mathbb{R}$ and $A=\{a, b\}$. Let $\Im$ be the topology on $X$ having the family $\{[-c, c]: c \in \mathbb{R}$ and $c>2\}$ as a base and let

$$
\tau=\{F \in S S(X, A): F(t) \in \Im \text { for every } t \in A\} .
$$

Then, $a_{0} \in \min (P O(X, \tau, A))$. On the other hand it is not difficult to check that $\min (X, \tau, A)=\emptyset$. Consider $G \in$ $S S(X, A)$, where $G(a)=[-2,2]$ and $G(b)=\emptyset$. Then $\left\{a_{x}: a_{x} \widetilde{\in} G\right\} \subseteq \min (P O(X, \tau, A))$ but $G \notin \min (X, \tau, A)$.

Theorem 3.17. Let $(X, \tau, A)$ be an STS and $a_{x} \in S P(X, A)$. Then $a_{x} \in \min (P O(X, \tau, A))$ if and only if $b_{y} \in \min (P O(X$, $\tau, A))$ for every $b_{y} \widetilde{\in} M_{\left(a_{x}, \tau\right)}$.

Proof. Necessity. Suppose that $a_{x} \in \min (P O(X, \tau, A))$ and let $b_{y} \tilde{\in} M_{\left(a_{x}, \tau\right)}$.

Claim. $a_{x} \widetilde{\in} C l_{\tau}\left(b_{y}\right)$.

Proof of Claim. Let $G \in \tau$ such that $a_{x} \tilde{\in} G$. Then $M_{\left(a_{x}, \tau\right)}$ $\widetilde{\subseteq} G$ and so $b_{y} \widetilde{\in} G$. Therefore, $b_{y}=b_{y} \widetilde{\cap} G$ and hence $b_{y} \widetilde{\cap} G \neq$ $0_{A}$. It follows that $a_{x} \widetilde{\in} C l_{\tau}\left(b_{y}\right)$.
Now by the above claim we have $C l_{\tau}\left(a_{x}\right) \widetilde{\simeq} C l_{\tau}\left(b_{y}\right)$. Since $a_{x} \in P O(X, \tau, A)$, then $a_{x} \widetilde{\subseteq} \operatorname{Int}_{\tau}\left(C l_{\tau}\left(a_{x}\right)\right)$ and so

$$
b_{y} \widetilde{\subseteq} M_{\left(a_{x}, \tau\right)} \widetilde{\widetilde{\subseteq}} \operatorname{Int}_{\tau}\left(C l_{\tau}\left(a_{x}\right)\right) \widetilde{\widetilde{\subseteq}} C l_{\tau}\left(a_{x}\right) \widetilde{\widetilde{\subseteq}} C l_{\tau}\left(b_{y}\right) .
$$

Hence, $b_{y} \in P O(X, \tau, A)$. Therefore, by Theorem 3.14, we have $b_{y} \in \min (P O(X, \tau, A))$.

Sufficiency. Suppose that $b_{y} \in \min (P O(X, \tau, A))$ for every $b_{y} \widetilde{\in} M_{\left(a_{x}, \tau\right)}$. Since $a_{x} \widetilde{\in} M_{\left(a_{x}, \tau\right)}$, then $a_{x} \in P O(X, \tau, A)$.

Therefore, by Theorem 3.14, we have

$$
a_{x} \in \min (P O(X, \tau, A)) .
$$

Corollary 3.18. Let $(X, \tau, A)$ be an STS. If $a_{x} \in \min (P O(X$, $\tau, A))$, then $M_{\left(a_{x}, \tau\right)} \in P O(X, \tau, A)$.

Theorem 3.19. Let $(X, \tau, A)$ be an STS. If $a_{x} \in \min (P O(X$, $\tau, A))$ and there exists $G \in \tau-\left\{0_{A}\right\}$ such that $G \widetilde{\subseteq} M_{\left(a_{x}, \tau\right)}$, then $M_{\left(a_{x}, \tau\right)}=G$.

Proof. Suppose that $a_{x} \in \min (P O(X, \tau, A))$ and $G \widetilde{\subseteq} M_{\left(a_{x}, \tau\right)}$ for some $G \in \tau-\left\{0_{A}\right\}$. Choose $b_{y} \widetilde{\in} G$, then $a_{x} \widetilde{\in} C l_{\tau}\left(b_{y}\right)$. Since $a_{x} \in P O(X, \tau, A)$, then there exists $F \in \tau$ such that $a_{x} \widetilde{\subseteq} F \widetilde{\subseteq} C l_{\tau}\left(a_{x}\right) \widetilde{\subseteq} C l_{\tau}\left(b_{y}\right)$. Since $a_{x} \widetilde{\in} F \in \tau$ and $a_{x} \widetilde{\in} C l_{\tau}\left(b_{y}\right)$, then $F \widetilde{\cap} b_{y} \neq 0_{A}$ and so $b_{y} \widetilde{\in} F \widetilde{\subseteq} C l_{\tau}\left(a_{x}\right)$. Since $b_{y} \widetilde{\in} G \in \tau$ and $b_{y} \tilde{\in} C l_{\tau}\left(a_{x}\right)$, then $G \widetilde{\cap} a_{x} \neq 0_{A}$ and hence $a_{x} \widetilde{\in} G$. It follows that $M_{\left(a_{x}, \tau\right)} \widetilde{\widetilde{\subseteq}} G$ and thus, $M_{\left(a_{x}, \tau\right)}=G$.

Corollary 3.20. Let $(X, \tau, A)$ be an STS. If $a_{x} \in \min (P O(X$, $\tau, A)$ ), then either $M_{\left(a_{x}, \tau\right)} \in \min (X, \tau, A)$ or $\operatorname{Int}_{\tau}\left(M_{\left(a_{x}, \tau\right)}\right)=$ $0_{A}$.

Theorem 3.21. Let $(X, \tau, A)$ be an STS and $a_{x} \in S S(X, A)$. Then the following are equivalent:

(a) $M_{\left(a_{x}, \tau\right)} \in \min (X, \tau, A)$.

(b) $a_{x} \in \min (P O(X, \tau, A))$ and $\operatorname{Int}_{\tau}\left(M_{\left(a_{x}, \tau\right)}\right) \neq 0_{A}$.

Proof. (a) $\Longrightarrow$ (b) Suppose that $M_{\left(a_{x}, \tau\right)} \in \min (X, \tau, A)$, then obviously that $\operatorname{Int}_{\tau}\left(M_{\left(a_{x}, \tau\right)}\right) \neq 0_{A}$. Also, by Theorem 3.15 we have $a_{x} \in \min (P O(X, \tau, A))$.

(b) $\Longrightarrow$ (a) Follows directly from Corollary 3.20.

\section{Soft Prehomogeneity}

The following is the main concept of this section:

Definition 4.1. An STS $(X, \tau, A)$ is called soft prehomogeneous if for any $a_{x}, b_{y} \in S P(X, A)$, there exists a soft prehomeomorphism $f_{p u}:(X, \tau, A) \longrightarrow(X, \tau, A)$ such that $f_{p u}\left(a_{x}\right)=b_{y}$. 
Theorem 4.2. For an STS $(X, \tau, A)$, the following are equivalent:

(a) $(X, \tau, A)$ is soft prehomogeneous.

(b) For any $a_{x}, b_{y} \in S P(X, A)$, there is a soft prehomeomorphism $f_{p u}:(X, \tau, A) \longrightarrow(X, \tau, A)$ such that $p(x)=y$ and $u(a)=b$.

(c) For any two pairs $(x, a),(y, b) \in X \times A$, there is a soft prehomeomorphism $f_{p u}:(X, \tau, A) \longrightarrow(X, \tau, A)$ such that $p(x)=y$ and $u(a)=b$.

Proof. Straightforward.

Remark 4.3. Soft homogeneous STSs are soft prehomogeneous.

Proof. Follows because soft homeomorphisms are soft prehomeomorphism.

Theorem 4.4. Every soft locally indiscrete STSs is soft prehomogeneous.

Proof. Let $(X, \tau, A)$ be a soft locally indiscrete STS. Let $c_{z}, d_{w} \in S P(X, A)$. Define $p: X \longrightarrow X$ and $u: A \longrightarrow A$ as follows:

$$
p(s)=\left\{\begin{array}{cc}
w & \text { if } s=z \\
z & \text { if } s=w \\
s & \text { if } s \neq z \text { and } s \neq w
\end{array}\right.
$$

and

$$
u(t)=\left\{\begin{array}{cc}
d & \text { if } t=c \\
c & \text { if } t=d \\
e & \text { if } t \neq c \text { and } t \neq d
\end{array} .\right.
$$

By Theorem 3.3, $P O(X, \tau, A)=S S(X, A)$ and thus $f_{p u}$ is a soft prehomeomorphism with $f_{p u}\left(a_{x}\right)=b_{y}$. Therefore, $(X, \tau, A)$ is soft prehomogeneous.

The following example shows that the converse of Remark 4.3 is not true, in general:

Example 4.5. Let $X=\mathbb{R}, A=\mathbb{Q}$. Let $F \in S S(X, A)$ defined by $F(a)=\mathbb{N}$ for every $a \in A$. Let $\tau=\left\{0_{A}, 1_{A}\right.$, $\left.F, 1_{A}-F\right\}$. Consider the STS $(X, \tau, A)$. Then $\tau=\tau^{c}$, and so $(X, \tau, A)$ is soft locally indiscrete. So by Theorem 4.4, $(X, \tau, A)$ is soft prehomogeneous. On the other hand, since $\min (X, \tau, A)=\left\{F, 1_{A}-F\right\}$ and $F(1)$ is countable but $\left(1_{A}-F\right)(1)$ is uncountable, then by Theorem 5.38(b) of [1], $(X, \tau, A)$ is not soft homogeneous.

The following is an example of a soft prehomogeneous STS that is neither soft homogeneous nor soft locally indiscrete:

Example 4.6. Let $X=\mathbb{N}, A=\{a, b\}$ and $\tau=\{F \in$
$S S(X, A): F(s) \in\{\{\emptyset\} \cup\{\{n, n+1, \ldots\}: n \in \mathbb{N}\}\}$ for all $s \in A\}$.

Then,

(1) $(X, \tau, A)$ is not soft locally indiscrete: Take $F \in S S(X, A)$ defined by $F(s)=\mathbb{N}-\{1\}$ for all $s \in A$. Then $F \in \tau-\tau^{c}$, and hence $(X, \tau, A)$ is not soft locally indiscrete.

(2) $(X, \tau, A)$ is not soft homogeneous: If $(X, \tau, A)$ is $(X, \tau, A)$, then by Theorem 5.14 of [1] the TS $\left(X, \tau_{a}\right)$ is homogeneous and so there is a homeomorphism $g:\left(X, \tau_{a}\right) \longrightarrow\left(X, \tau_{a}\right)$ such that $g(1)=2$. But $\{1\} \in\left(\tau_{a}\right)^{c}$ while $g(\{1\})=\{2\} \notin\left(\tau_{a}\right)^{c}$. It follows that $(X, \tau, A)$ is not soft homogeneous.

(3) $(X, \tau, A)$ is soft prehomogeneous: Let $c_{x}, d_{y} \in S P(X, A)$. Define $p: X \longrightarrow X$ and $u: A \longrightarrow A$ as follows:

$$
p(s)=\left\{\begin{array}{cc}
y & \text { if } s=x \\
x & \text { if } s=y \\
s & \text { if } s \neq x \text { and } s \neq y
\end{array}\right.
$$

and

$$
u(t)=\left\{\begin{array}{cc}
d & \text { if } t=c \\
c & \text { if } t=d \\
e & \text { if } t \neq c \text { and } t \neq d
\end{array} .\right.
$$

It is not difficult to check that $P O(X, \tau, A)=\{F \in S S(X$, $A): F(s) \in\{\emptyset, \mathbb{N}\}\} \cup\{F \in S S(X, A): F(a)$ and $F(b)$ are infinite sets $\}$, and thus $f_{p u}$ is a soft prehomeomorphism with $f_{p u}\left(c_{x}\right)=d_{y}$. Hence, $(X, \tau, A)$ is soft prehomogeneous.

Theorem 4.7. Let $(X, \tau, A)$ be an STS with $\min (P O(X, \tau$, $A)) \neq \emptyset$. Then $(X, \tau, A)$ is soft prehomogeneous if and only if $(X, \tau, A)$ is soft locally indiscrete.

Proof. Necessity. Suppose that $(X, \tau, A)$ is soft prehomogeneous. We will apply Theorem 3.3(c). Let $b_{y} \in S P(X, A)$. Pick $a_{x} \in \min (P O(X, \tau, A))$. By soft prehomogeneity of $(X, \tau, A)$, there exists a soft prehomeomorphism $f_{p u}:(X, \tau, A)$ $\longrightarrow(X, \tau, A)$ such that $f_{p u}\left(a_{x}\right)=b_{y}$ and so $b_{y} \in P O(X, \tau$, A).

Sufficiency. Follows from Theorem 4.4.

Corollary 4.8. Let $(X, \tau, A)$ be an STS with $\min (X, \tau, A) \neq$ $\emptyset$. Then $(X, \tau, A)$ is soft prehomogeneous if and only if $(X, \tau, A)$ is soft locally indiscrete.

Proof. Follows from Theorems 3.15 and 4.7.

Corollary 4.9. Let $(X, \tau, A)$ be an STS such that $\tau$ is finite. Then $(X, \tau, A)$ is soft prehomogeneous if and only if $(X, \tau, A)$ is soft locally indiscrete.

Proof. Since $\tau$ is finite, then it is easy to see that $\min (X, \tau$, 
$A) \neq \emptyset$. Thus, by Corollary 4.8 we get the result.

In the following result, $\tau_{p r}$ denotes the soft topology on $X$ relative to $A$ having $P O(X, \tau, A)$ as a subbase.

Theorem 4.10. If $(X, \tau, A)$ is soft prehomogeneous, then $\left(X, \tau_{p r}, A\right)$ is soft homogeneous.

Proof. Suppose that $(X, \tau, A)$ is soft prehomogeneous and let $a_{x}, b_{y} \in S S(X, A)$. Since $(X, \tau, A)$ is soft prehomogeneous, there exists a soft prehomeomorphism $f_{p u}:(X, \tau, A)$ $\longrightarrow(X, \tau, A)$ such that $f_{p u}\left(a_{x}\right)=b_{y}$. Let $G$ be a soft basic soft open set in $\left(X, \tau_{p r}, A\right)$, then $G=\widetilde{\bigcap_{i=1}^{n}} G_{i}$ where $G_{i} \in P O(X, \tau, A)$ for all $i=1,2, \ldots, n$. Now, for every $i \in\{1,2, \ldots, n\}, f_{p u}^{-1}\left(G_{i}\right) \in P O(X, \tau, A)$ and so $f_{p u}^{-1}(G)=$ $\widetilde{n} f_{p u}^{-1}\left(G_{i}\right) \in \tau_{p r}$. Therefore, $f_{p u}:\left(X, \tau_{p r}, A\right) \longrightarrow(X$, $\left.\tau_{p r}, A\right)$ is soft continuous. By a similar way we can see that $f_{p^{-1} u^{-1}}$ is soft continuous. Therefore, $f_{p u}:\left(X, \tau_{p r}, A\right) \longrightarrow$ $\left(X, \tau_{p r}, A\right)$ is a soft homeomorphism with $f_{p u}\left(a_{x}\right)=b_{y}$. Hence, $\left(X, \tau_{p r}, A\right)$ is soft homogeneous.

By the end of this section, we raise the following question regarding the converse of Theorem 4.10:

Question 4.11. Let $(X, \tau, A)$ be an STS such that $\left(X, \tau_{p r}, A\right)$ is soft homogeneous. Is it true that $(X, \tau, A)$ is soft prehomogeneous?

\section{Relative TSs and Generated STSs}

Theorem 5.1. Let $(X, \tau, A)$ be an STS. Then for any $a_{x} \in$ $S P(X, A), M_{\left(a_{x}, \tau\right)}(a)=M_{\left(x, \tau_{a}\right)}$.

Proof. Let $a_{x} \in S P(X, A)$, then

$$
\begin{aligned}
M_{\left(a_{x}, \tau\right)}(a) & =\left(\widetilde{\cap}\left\{F: F \in \tau \text { and } a_{x} \widetilde{\in} F\right\}\right)(a) \\
& =\cap\left\{F(a): F \in \tau \text { and } a_{x} \widetilde{\in} F\right\} \\
& =(\cap\{F(a): F \in \tau \text { and } x \in F(a)\})(a) \\
& =\cap\left\{U \in \tau_{a}: x \in U\right\} \\
& =M_{\left(x, \tau_{a}\right) .}
\end{aligned}
$$

Lemma 5.2. Let $(X, \tau, A)$ be an STS and let $\mathcal{B}$ be a soft base of $(X, \tau, A)$. Then for any $a_{x} \in S P(X, A), M_{\left(a_{x}, \tau\right)}=$ $\widetilde{\cap}\left\{B \in \mathcal{B}: a_{x} \widetilde{\in} B\right\}$.

Proof. Let $a_{x} \in S P(X, A)$, then clearly that

$$
M_{\left(a_{x}, \tau\right)} \widetilde{\widetilde{\subseteq}} \widetilde{\cap}\left\{B \in \mathcal{B}: a_{x} \widetilde{\in} B\right\} .
$$

Conversely, let $b_{y} \widetilde{\in} \widetilde{\cap}\left\{B \in \mathcal{B}: a_{x} \widetilde{\in} B\right\}$ and let $G \in \tau$ such that $a_{x} \widetilde{\in} G$. Choose $B \in \mathcal{B}$ such that $a_{x} \widetilde{\in} B \widetilde{\subseteq} G$, then $b_{y} \widetilde{\in} B \widetilde{\subseteq} G$. It follows that $b_{y} \widetilde{\in} M_{\left(a_{x}, \tau\right)}$.

Theorem 5.3. Let $X$ be an initial universe and let $A$ be a set of parameters. Let $\left\{\Im_{a}: a \in A\right\}$ be an indexed family of topologies on $X$ and let $\sigma=\bigoplus_{a \in A} \Im_{a}$. Then for every $a_{x} \in$ $\operatorname{SP}(X, A), M_{\left(a_{x}, \sigma\right)}=a_{M_{\left(x, \Im_{a}\right)}}$.

Proof. By Theorem 3.5 of [1], $\left\{b_{Y}: b \in A\right.$ and $\left.Y \in \Im_{b}\right\}$ is a soft base of $(X, \sigma, A)$. So, by Lemma 5.2,

$$
\begin{aligned}
M_{\left(a_{x}, \sigma\right)} & =\widetilde{\cap}\left\{b_{Y}: b \in A, Y \in \Im_{b} \text { and } a_{x} \widetilde{\in} b_{Y}\right\} \\
& =\widetilde{\cap}\left\{a_{Y}: Y \in \Im_{a} \text { and } x \in Y\right\} \\
& =a_{\left\{Y: Y \in \Im_{a} \text { and } x \in Y\right\}} \\
& =a_{M_{\left(x, \Im_{a}\right)} .}
\end{aligned}
$$

Corollary 5.4. Let $X$ be an initial universe and let $A$ be a set of parameters. Let $\left\{\Im_{a}: a \in A\right\}$ be an indexed family of topologies on $X$. Then

$$
\operatorname{minss}(X, \tau, A)=\left\{a_{Y}: a \in A \text { and } Y \in \min s\left(X, \Im_{a}\right)\right\} .
$$

Lemma 5.5. Let $(X, \tau, A)$ be an STS. Then for every $a_{x} \in$ $S P(X, A),\left(C l_{\tau}\left(a_{x}\right)\right)(a)=C l_{\tau_{a}}(\{x\})$.

Proof. By Proposition 7 of [4], $C l_{\tau_{a}}(\{x\}) \subseteq\left(C l_{\tau}\left(a_{x}\right)\right)(a)$. To show that $\left(C l_{\tau}\left(a_{x}\right)\right)(a) \subseteq C l_{\tau_{a}}(\{x\})$, suppose to the contrary that there exists $y \in\left(C l_{\tau}\left(a_{x}\right)\right)(a)-C l_{\tau_{a}}(\{x\})$. Since $y \notin C l_{\tau_{a}}(\{x\})$, then there exists $V \in \tau_{a}$ such that $y \in V$ and $x \notin V$. Choose $F \in \tau$ such that $F(a)=V$. Then $a_{x} \widetilde{\in} 1_{A}-F$. On the other hand, since we have $a_{y} \widetilde{\in} F \in \tau$ and $a_{y} \widetilde{\in} C l_{\tau}\left(a_{x}\right)$, then $a_{x} \widetilde{\in} F$, a contradiction.

Theorem 5.6. Let $(X, \tau, A)$ be an STS. If $a_{x} \in$ $\min (P O(X, \tau, A))$, then $\{x\} \in \min \left(P O\left(X, \tau_{a}\right)\right)$.

Proof. Suppose that $a_{x} \in \min (P O(X, \tau, A))$. Then $a_{x} \in$ $P O(X, \tau, A)$ and so there exists $F \in \tau$ such that $a_{x} \widetilde{\subseteq} F \widetilde{\subseteq} C l_{\tau}$ $\left(a_{x}\right)$. Thus we have $F(a) \in \tau_{a}$ and $\{x\} \subseteq F(a) \subseteq\left(C l_{\tau_{a}}\left(a_{x}\right)\right)$ (a). On the other hand, by Lemma 5.5, $\left(C l_{\tau}\left(a_{x}\right)\right)(a)=$ $C l_{\tau_{a}}(\{x\})$. Hence, $\{x\} \in P O\left(X, \tau_{a}\right)$. Therefore, $\{x\} \in$ $\min \left(P O\left(X, \tau_{a}\right)\right)$.

The following lemma will be used in the following result:

Lemma 5.7. Let $X$ be an initial universe and let $A$ be a set of parameters. Let $\left\{\Im_{a}: a \in A\right\}$ be an indexed family of topologies on $X$ and let $\sigma=\bigoplus_{a \in A} \Im_{a}$. Then for any $G \in$ 
$S S(X, A)$ and any $a \in A, C l_{\Im_{a}}(G(a))=\left(C l_{\sigma}(G)\right)(a)$.

Proof. Let $G \in S S(X, A)$ and $a \in A$. By Theorem 3.7 of [1], $\sigma_{a}=\Im_{a}$. So by Proposition 7 of [4], $C l_{\Im_{a}}(G(a)) \subseteq$ $\left(C l_{\sigma}(G)\right)(a)$. To show that $\left(C l_{\sigma}(G)\right)(a) \subseteq C l_{\Im_{a}}(G(a))$, let $x \in\left(C l_{\sigma}(G)\right)(a)$ and let $U \in \Im_{a}$ such that $x \in U$. Since $a_{x} \widetilde{\in} a_{U}$ and $a_{x} \widetilde{\in} C l_{\sigma}(G)$, then $G \widetilde{\cap} a_{U} \neq 0_{A}$. Thus, $\left(G \widetilde{\cap} a_{U}\right)(a)=G(a) \cap U \neq \emptyset$. It follows that $x \in C l_{\Im}(G(a))$.

Theorem 5.8. Let $X$ be an initial universe and let $A$ be a set of parameters. Let $\left\{\Im_{a}: a \in A\right\}$ be an indexed family of topologies on $X$ and let $\sigma=\bigoplus_{a \in A} \Im_{a}$. Then $G \in P O(X, \sigma, A)$ if and only if $G(a) \in P O\left(X, \Im_{a}\right)$ for all $a \in A$.

Proof. Necessity. Suppose that $G \in P O(X, \sigma, A)$ and let $a \in A$. Choose $F \in \sigma$ such that $G \widetilde{\subseteq} F \widetilde{\subseteq} C l_{\sigma}(G)$. Then by Lemma 5.7, $G(a) \subseteq F(a) \subseteq\left(C l_{\sigma}(G)\right)(a)=C l_{\Im_{a}}(G(a))$. Since $F \in \sigma$, then by Theorem 3.7 of [1], $F(a) \in \Im_{a}$. It follows that $G(a) \in P O\left(X, \Im_{a}\right)$.

Sufficiency. Suppose that $G(a) \in P O\left(X, \Im_{a}\right)$ for all $a \in A$. For each $a \in A$, choose $U_{a} \in \Im_{a}$ such that $G(a) \subseteq U_{a} \subseteq$ $C l_{\Im_{a}}(G(a))$. Let $F \in S S(X, A)$ defined by $F(a)=U_{a}$ for all $a \in A$. Then $F \in \sigma$ and by Lemma 5.7, $G(a) \subseteq F(a) \subseteq$ $C l_{\Im_{a}}(G(a))=\left(C l_{\sigma}(G)\right)(a)$ for all $a \in A$ which implies that $G \widetilde{\subseteq} F \widetilde{\subseteq} C l_{\sigma}(G)$. Hence, $G \in P O(X, \sigma, A)$.

Corollary 5.9. Let $(X, \Im)$ be a TS and $A$ be a set of parameters. Then $G \in P O(X, \tau(\Im), A)$ if and only if $G(a) \in$ $P O(X, \Im)$ for all $a \in A$.

Proof. For every $a \in A$, put $\Im_{a}=\Im$. Then $\tau(\Im)=\bigoplus_{a \in A} \Im_{a}$. So by Theorem 5.8, we get the result.

Theorem 5.10. Let $X$ be an initial universe and $A$ be a set of parameters. Let $\left\{\Im_{a}: a \in A\right\}$ be an indexed family of topologies on $X$. Then $\min \left(P O\left(X, \bigoplus_{a \in A} \Im_{a}, A\right)\right)=\left\{a_{x}\right.$ : $a \in A$ and $\left.\{x\} \in \min \left(P O\left(X, \Im_{a}\right)\right)\right\}$.

Proof. Let $G \in \min \left(P O\left(X, \bigoplus_{a \in A} \Im_{a}, A\right)\right)$, then by Theorem 3.14 there exists $a_{x} \in P O\left(X, \bigoplus_{a \in A} \Im_{a}, A\right) \cap S P(X, A)$ such that $G=a_{x}$. By Theorem 3.7 of [1] and Theorem 5.6, we have $\{x\} \in \min \left(P O\left(X, \Im_{a}\right)\right)$. This ends the proof that $\min \left(P O\left(X, \bigoplus_{a \in A} \Im_{a}, A\right)\right) \subseteq\left\{a_{x}: a \in A\right.$ and $\{x\} \in$ $\left.\min \left(P O\left(X, \Im_{a}\right)\right)\right\}$.

Conversely, let $a \in A$ and $\{x\} \in \min \left(P O\left(X, \Im_{a}\right)\right)$. Then

$$
\left(a_{x}\right)(b)= \begin{cases}\{x\}, & \text { if } b=a, \\ \emptyset, & \text { if } b \neq a\end{cases}
$$

and so $\left(a_{x}\right)(b) \in P O\left(X, \Im_{a}\right)$ for all $a \in A$. Thus by Theorem 5.8, $a_{x} \in P O\left(X, \bigoplus_{a \in A} \Im_{a}, A\right)$. Therefore, by Theorem 3.14, we have $a_{x} \in \min \left(P O\left(X, \bigoplus_{a \in A} \Im_{a}, A\right)\right)$.

Corollary 5.11. Let $X$ be an initial universe and $A$ be a set of parameters. Then $\min (P O(X, \tau(\Im), A))=\left\{a_{x}: a \in A\right.$ and $\{x\} \in \min (P O(X, \Im))\}$.

Proof. For every $a \in A$, put $\Im_{a}=\Im$. Then $\tau(\Im)=\bigoplus_{a \in A} \Im_{a}$. So by Theorem 5.10, we get the result.

Lemma 5.12. If $f_{p u}:\left(X, \bigoplus_{a \in A} \Im_{a}, A\right) \longrightarrow\left(X, \bigoplus_{a \in A} \Im_{a}, A\right)$ is soft preirresolute with $u(b)=b$, then $p:\left(X, \Im_{b}\right) \longrightarrow$ $\left(X, \Im_{b}\right)$ is preirresolute.

Proof. Let $U \in P O\left(X, \Im_{b}\right)$. Then by Theorem 5.8, $b_{U} \in$ $P O\left(X, \bigoplus_{a \in A} \Im_{a}, A\right)$ and so, $f_{p u}^{-1}\left(b_{U}\right) \in P O\left(X, \bigoplus_{a \in A} \Im_{a}, A\right)$. So again by Theorem 5.8, $\left(f_{p u}^{-1}\left(b_{U}\right)\right)(b) \in P O\left(X, \Im_{b}\right)$. But $\left(f_{p u}^{-1}\left(b_{U}\right)\right)(b)=p^{-1}\left(b_{U}\left(u^{-1}(b)\right)=p^{-1}(U)\right.$. Therefore, $p:\left(X, \Im_{b}\right) \longrightarrow\left(X, \Im_{b}\right)$ is preirresolute.

Theorem 5.13. Let $X$ be an initial universe and $A$ be a set of parameters. Let $\left\{\Im_{a}: a \in A\right\}$ be an indexed family of topologies on $X$. If $\left(X, \bigoplus_{a \in A} \Im_{a}, A\right)$ is soft prehomogeneous, then for every $b \in A,\left(X, \Im_{b}\right)$ is prehomogeneous.

Proof. Suppose that $\left(X, \bigoplus_{a \in A} \Im_{a}, A\right)$ is soft prehomogeneous and let $b \in A$. Let $x, y \in X$, then $b_{x}, b_{y} \in S P(X, A)$ and so there exists a soft prehomeomorphism $f_{p u}:\left(X, \bigoplus_{a \in A} \Im_{a}\right.$, $A) \longrightarrow\left(X, \bigoplus_{a \in A} \Im_{a}, A\right)$ such that $f_{p u}\left(b_{x}\right)=b_{y}$. Then $p:$ $X \longrightarrow X$ is a bijection with $p(x)=y$. Since $u(b)=$ $u^{-1}(b)=b$, then by Lemma 5.12, the functions $p, p^{-1}$ : $\left(X, \Im_{b}\right) \longrightarrow\left(X, \Im_{b}\right)$ are preirresolute. It follows that $p:$ $\left(X, \Im_{b}\right) \longrightarrow\left(X, \Im_{b}\right)$ is a prehomeomorphism with $p(x)=y$ Hence, $\left(X, \Im_{b}\right)$ is prehomogeneous.

Question 5.14. Let $\left\{\left(X, \Im_{a}\right): a \in A\right\}$ be an indexed family of prehomogeneous TSs. Is it true that $\left(X, \bigoplus_{a \in A} \Im_{a}, A\right)$ is soft prehomogeneous.

The following result gives a partial answer for Question 5.14:

Theorem 5.15. Let $(X, \Im)$ be a TS and $A$ be a set of parameters. Then $(X, \tau(\Im), A)$ is soft prehomogeneous if and only if $(X, \Im)$ is prehomogeneous.

Proof. Necessity. Suppose that $(X, \tau(\Im), A)$ is soft prehomogeneous. For every $b \in A$, put $\Im_{b}=\Im$. Then $\tau(\Im)=$ 
$\bigoplus_{b \in A} \Im_{b}$. Thus, by Theorem 5.13, we get the result.

Sufficiency. Suppose that $(X, \Im)$ is prehomogeneous. Let $a_{x}, b_{y} \in S P(X, A)$, then there is a prehomeomorphism $p:$ $(X, \Im) \longrightarrow(X, \Im)$ such that $p(x)=y$. Choose a bijection $u$ : $A \longrightarrow A$ such that $u(a)=b$. Then $f_{p u}:(X, \tau(\Im), A) \longrightarrow$ $(X, \tau(\Im), A)$ is a bijection. To show that $f_{p u}$ is soft preirresolute, let $G \in P O(X, \tau(\Im), A)$, then by Corollary 5.9, $G(c) \in P O(X, \Im)$ for all $c \in A$. Since $p:(X, \Im) \longrightarrow$ $(X, \Im)$ is preirresolute, then for every $d \in A,\left(f_{p u}^{-1}(G)\right)(d)=$ $p^{-1}\left(G\left(u^{-1}(d)\right)\right) \in P O(X, \Im)$. Therefore, again by Corollary 5.9, $f_{p u}^{-1}(G) \in P O(X, \tau(\Im), A)$. Hence, $f_{p u}:(X, \tau(\Im), A)$ $\longrightarrow(X, \tau(\Im), A)$ is soft preirresolute. Similarly, we can show that $f_{p^{-1} u^{-1}}:(X, \tau(\Im), A) \longrightarrow(X, \tau(\Im), A)$ is soft preirresolute. It follows that $(X, \tau(\Im), A)$ is soft prehomogeneous.

The following three questions are natural:

Question 5.16. Let $f_{p u}:(X, \tau, A) \longrightarrow(X, \tau, A)$ be soft prehomeomorphism and $a \in A$. Is it true that $p:\left(X, \tau_{a}\right) \longrightarrow$ $\left(X, \tau_{a}\right)$ is a prehomeomorphism?

Question 5.17. Let $(X, \tau, A)$ be soft prehomogeneous and $a \in A$. Is it true that $\left(X, \tau_{a}\right)$ is prehomogeneous?

Question 5.18. Let $\left(X, \tau_{a}\right)$ be prehomogeneous for all $a \in$ $A$. Is it true that $(X, \tau, A)$ is soft prehomogeneous?

We will leave Questions 5.16 and 5.17 as open questions. On the other hand, the following example gives a negative answer for Question 5.18:

Example 5.19. Let $X=\mathbb{R}$ and $A=\{a, b\}$. Let

$$
\begin{aligned}
& F=\{(a,(-\infty, 0)),(b,(-\infty, 1))\} \\
& G=\{(a,[0,1)),(b,(-\infty, 1))\} \\
& H=\{(a,[1,2)),(b,[1, \infty))\} \\
& K=\{(a,[2, \infty)),(b,[1, \infty))\} \\
& L=\{(a, \emptyset),(b,(-\infty, 1))\} \\
& M=\{(a, \emptyset),(b,[1, \infty))\}
\end{aligned}
$$

Let $\mathcal{M}=\{F, G, H, K, L, M\}$. Consider the STS $(X, \tau, A)$, where $\tau$ having $\mathcal{M}$ as a soft base. Then $\tau_{a}$ is the topology on $X$ having $\{(-\infty, 0),[0,1),[1,2),[2, \infty)\}$ as a base, and $\tau_{b}$ is the topology on $X$ having $\{(-\infty, 1),[1, \infty)\}$ as a base. Hence, $\left(X, \tau_{a}\right)$ and $\left(X, \tau_{b}\right)$ are both locally indiscrete. On the other hand, it is clear that $1_{A}-F \notin \tau$ and hence $(X, \tau, A)$ is not soft locally indiscrete. Therefore, by Corollary $4.9,(X, \tau, A)$ is not soft prehomogeneous.

\section{Conclusion}

Several characterizations of soft minimal soft open sets are introduced, and two new kinds of soft sets are introduced and investigated. Moreover, the notion of soft prehomogeneity is introduced and investigated. Several relationships are studied. Also, some characterizations of soft locally indiscrete are introduced. Six open questions are raised. We expect that our results will be important for the forthcoming in SBTSs to build a good background for some practical applications and to answer the most intricate problems containing uncertainty in medical, engineering, economics, environment, and in general. In future studies, the following topics could be considered: (1) defining soft prehomogeneity components; and (2) defining soft semihomogeneity.

\section{Conflict of Interest}

No potential conflict of interest relevant to this article was reported.

\section{References}

[1] S. Al Ghour and A. Bin-Saadon, "On some generated soft topological spaces and soft homogeneity," Heliyon, vol. 5, no. 7, article no. e02061, 2019. https://doi.org/10.1016/j. heliyon.2019.e02061

[2] S. Al Ghour and W. Hamed, "On two classes of soft sets in soft topological spaces," Symmetry, vol. 12, no. 2, article no. 265,2020 . https://doi.org/10.3390/sym12020265

[3] D. Molodtsov, "Soft set theory: first results," Computers \& Mathematics with Applications, vol. 37, no. 4-5, pp. 19-31, 1999. https://doi.org/10.1016/S0898-1221(99)00056-5

[4] M. Shabir and M. Naz, "On soft topological spaces," Computers \& Mathematics with Applications, vol. 61, no. 7, pp. 1786-1799, 2011. https://doi.org/10.1016/j.camwa.2011. 02.006

[5] S. Alkhazaleh and E. A. Marei, "New soft rough set approximations," International Journal of Fuzzy Logic and Intelligent Systems, vol. 21, no. 2, pp. 123-134, 2021. https://doi.org/10.5391/IJFIS.2021.21.2.123

[6] S. Al Ghour, "Strong form of soft semi-open sets in soft topological spaces," International Journal of Fuzzy Logic 
and Intelligent Systems, vol. 21, no. 2, pp. 159-168, 2021. https://doi.org/10.5391/IJFIS.2021.21.2.159

[7] S. Al Ghour, "Soft $\omega *$-paracompactness in soft topological spaces," International Journal of Fuzzy Logic and Intelligent Systems, vol. 21, no. 1, pp. 57-65, 2021. https://doi.org/10.5391/IJFIS.2021.21.1.57

[8] T. M. Al-Shami, "New soft structure: infra soft topological spaces," Mathematical Problems in Engineering, vol. 2021, article no. 3361604, 2021. https://doi.org/10.1155/ 2021/3361604

[9] T. M. Al-Shami, "Infra soft compact spaces and application to fixed point theorem," Journal of Function Spaces, vol. 2021, article no. 3417096, 2021. https://doi.org/10. 1155/2021/3417096

[10] T. M. Al-Shami, "Homeomorphism and quotient mappings in infrasoft topological spaces," Journal of Mathematics, vol. 2021, article no. 3388288, 2021. https: //doi.org/10.1155/2021/3388288

[11] T. M. Al-Shami and E. S. A. Abo-Tabl, "Connectedness and local connectedness on infra soft topological spaces," Mathematics, vol. 9, no. 15, article no. 1759, 2021. https: //doi.org/10.3390/math9151759

[12] R. Mousarezaei and B. Davvaz, "On soft topological polygroups and their examples," International Journal of Fuzzy Logic and Intelligent Systems, vol. 21, no. 1, pp. 29-37, 2021. https://doi.org/10.5391/IJFIS.2021.21.1.29

[13] S. Oztunç, S. Aslan, and H. Dutta, "Categorical structures of soft groups," Soft Computing, vol. 25, no. 4, pp. 30593064, 2021. https://doi.org/10.1007/s00500-020-05362-0

[14] T. M. Al-Shami and E. A. Abo-Tabl, "Soft a-separation axioms and a-fixed soft points," AIMS Mathematics, vol. 6, no. 6, pp. 5675-5694, 2021. https://doi.org/10.3934/ math.2021335

[15] T. M. Al-Shami, "On soft separation axioms and their applications on decision-making problem," Mathematical Problems in Engineering, vol. 2021, article no. 8876978, 2021. https://doi.org/10.1155/2021/8876978

[16] T. M. Al-Shami, "Bipolar soft sets: relations between them and ordinary points and their applications," Complexity, vol. 2021, article no. 6621854, 2021. https://doi. org/10.1155/2021/6621854
[17] T. M. Al-Shami, I. Alshammari, and B. A. Asaad, "Soft maps via soft somewhere dense sets," Filomat, vol. 34, no. 10, pp. 3429-3440, 2020. https://doi.org/10.2298/ FIL2010429A

[18] G. Oguz, "Soft topological transformation groups," Mathematics, vol. 8, no. 9, article no. 1545, 2020. https: //doi.org/10.3390/math8091545

[19] H. S. Al-Saadi and W. K. Min, "On soft generalized closed sets in a soft topological space with a soft weak structure," International Journal of Fuzzy Logic and Intelligent Systems, vol. 17, no. 4, pp. 323-328, 2017. https://doi.org/10.5391/IJFIS.2017.17.4.323

[20] V. Cetkin, E. Guner, and H. Aygun, "On 2S-metric spaces," Soft Computing, vol. 24, no. 17, pp. 12731-12742, 2020. https://doi.org/10.1007/s00500-020-05134-w

[21] M. E. El-Shafei and T. M. Al-Shami, "Applications of partial belong and total non-belong relations on soft separation axioms and decision-making problem," Computational and Applied Mathematics, vol. 39, article no. 138, 2020. https://doi.org/10.1007/s40314-020-01161-3

[22] J. C. R. Alcantud, "Soft open bases and a novel construction of soft topologies from bases for topologies," Mathematics, vol. 8, no. 5, article no. 672, 2020. https: //doi.org/10.3390/math8050672

[23] A. A. Bahredar and N. Kouhestani, "On $\varepsilon$-soft topological semigroups," Soft Computing, vol. 24, no. 10, pp. 70357046, 2020. https://doi.org/10.1007/s00500-020-04826-7

[24] T. M. Al-Shami and M. E. El-Shafei, "Partial belong relation on soft separation axioms and decision-making problem, two birds with one stone," Soft Computing, vol. 24, no. 7, pp. 5377-5387, 2020. https://doi.org/10.1007/ s00500-019-04295-7

[25] T. M. Al-Shami, L. D. Kocinac, and B. A. Asaad, "Sum of soft topological spaces," Mathematics, vol. 8, no. 6, article no. 990, 2020. https://doi.org/10.3390/math8060990

[26] T. M. Al-Shami, "Soft somewhere dense sets on soft topological spaces," Communications of the Korean Mathematical Society, vol. 33, no. 4, pp. 1341-1356, 2018. https://doi.org/10.4134/CKMS.c170378

[27] F. Nakaoka and N. Oda, "Some applications of minimal open sets," International Journal of Mathematics and 
Mathematical Sciences, vol. 27, article no. 784531, 2001. https://doi.org/10.1155/S0161171201006482

[28] R. A. H. Al-Abdulla, "On strong and weak sets in topological spaces," Journal of Interdisciplinary Mathematics, vol. 24, no. 3, pp. 765-773, 2021. https://doi.org/10.1080/ 09720502.2021 .1884391

[29] E. R. R. Rodriguez and S. Namiq, "On minimal $\lambda$ co-open sets," Proyecciones, vol. 39, no. 2, pp. 421-433, 2020. https://doi.org/10.22199/issn.0717-6279-2020-02-0026

[30] E. Rosas and S. F. Namiq, "On minimal $\lambda$ rc-open sets," Italian Journal of Pure and Applied Mathematics, vol. 43, pp. 868-877, 2020.

[31] K. B. Bagchi and A. Mukharjee, "On maximal, minimal and mean open sets," Afrika Matematika, vol. 30, pp. 291296, 2019. https://doi.org/10.1007/s13370-018-0644-7

[32] S. Al Ghour, "Minimality and prehomogeneity," Acta Mathematica Universitatis Comenianae, vol. 72, no. 2, pp. 237-244, 2003.

[33] K. Naganagouda and C. Chetana, "Soft minimal open sets and soft maximal open sets in soft topological spaces," $J$. Comput. Math. Sci., vol. 4, no. 2, pp. 149-155, 2015.

[34] A. S. Mashhour, "On preconlinuous and weak precontinuous mappings," Proceedings of the Mathematical and Physical Society of Egypt, vol. 53, pp. 47-53, 1982.

[35] P. K. Maji, R. Biswas, and A. R. Roy, "Soft set theory," Computers \& Mathematics with Applications, vol. 45, no. 4-5, pp. 555-562, 2003. https://doi.org/10.1016/S08981221(03)00016-6
[36] I. Zorlutuna, M. Akdag, W. K. Min, and S. Atmaca, "Remarks on soft topological spaces," Annals of Fuzzy Mathematics and Informatics, vol. 3, no. 2, pp. 171-185, 2012.

[37] S. Das and S. K. Samanta, "Soft metric," Annals of Fuzzy Mathematics and Informatics, vol. 6, no. 1, pp. 77-94, 2013.

[38] M. Terepeta, "On separating axioms and similarity of soft topological spaces," Soft Computing, vol. 23, no. 3, pp. 1049-1057, 2019. https://doi.org/10.1007/s00500-0172824-z

[39] I. Arockiarani and A. Lancy, "Generalized soft g $\beta$-closed sets and soft gs $\beta$-closed sets in soft topological spaces," International Journal of Mathematical Archive, vol. 4, no. 2, pp. 1-7, 2013.

[40] I. Arockiarani and A. Selvi, "On soft slightly $\pi \mathrm{g}$ continuous functions," Journal of Progressive Research in Mathematics, vol. 3, no. 2, pp. 168-174, 2015.

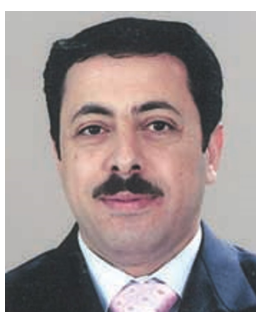

Samer Al Ghour received the Ph.D. in Mathematics from University of Jordan, Jordan in 1999. Currently, he is a professor at the Department of Mathematics and Statistics, Jordan University of Science and Technology, Jordan. His research interests is include general topology, fuzzy topology, and soft set theory.

E-mail: algore@just.edu.jo 